\title{
G-TRANSVERSALITY REVISITED
}

\author{
S. R. COSTENOBLE AND S. WANER \\ (Communicated by Frederick R. Cohen)
}

\begin{abstract}
We express the obstruction to $G$-transversality in two related ways: as an element in the quotient of homotopical equivariant normal bordism by geometric bordism and also as the obstruction in a homotopy lifting problem. In both cases, our formulation is given in terms of the initial data only, in contrast to earlier theories. We also obtain related obstructions to equivariant "near" transversality, i.e., transversality using arbitrarily small homotopies.
\end{abstract}

\section{INTRODUCTION}

The $G$-transversality problem is this: Given smooth $G$-manifolds $M$ and $N$, a smooth $G$-invariant submanifold $Y \subset N$, and a $G$-map $f: M \rightarrow N$, is $f$ $G$-homotopic to a map that is transverse to $Y$ ? As is well known, the answer may be no, in contrast to the nonequivariant case. Further, this is a global problem in the sense that if $f$ is $G$-homotopic to a transverse map then the homotopy may not be a small one, in contrast again to the nonequivariant case. If we require that it be possible to make the $G$-homotopy arbitrarily small, we get the different problem of "near" $G$-transversality.

Wasserman [Was] gives very useful sufficient conditions for $G$-transversality to hold and Petrie [P] gives an obstruction theory. These discussions, however, are not all that can be said about the $G$-transversality problem, for the following reasons. First, Petrie's obstructions and obstruction groups are not well defined, in the sense that they are not given in terms of the initial data only. Second, whereas Petrie mentions that the $G$-transversality problem is a global one, neither Petrie's nor Wasserman's discussion distinguishes near transversality from the global theory.

We present here two related approaches to the $G$-transversality problem for a finite transformation group $G$. In the first we restrict attention to the case where $N$ is the Thom space of a bundle over $Y$ and consider the following stable form of the problem which arises in the study of equivariant Poincare complexes [CW]: If $W$ is a finite-dimensional orthogonal $G$-module, let $D(W)$ denote the unit disc in $W$ and $S(W)$ its boundary. The stable problem is then: Given a $G$-map $f: M \rightarrow N$ of smooth $G$-manifolds and $Y \subset N$ a smooth $G$ invariant submanifold, is there a $W$ such that $f \times 1: M \times D(W) \rightarrow N \times D(W)$

Received by the editors February 27, 1991.

1991 Mathematics Subject Classification. Primary 57R91; Secondary 55S91, 55N91, 57R85. 
is $G$-homotopic rel $M \times S(W)$ to a map that is transverse to $Y \times 0$ ? We display the obstruction to stable $G$-transversality as an element of $\underline{\Omega} / \Omega$, where $\Omega$ is geometric equivariant normal cobordism and $\underline{\Omega}$ is homotopical equivariant normal cobordism. This shows that the failure of $G$-transversality is not only responsible for, but is equivalent to, the dichotomy between geometric and homotopical equivariant cobordism studied in [tD] and elsewhere.

Our second approach is to translate the $G$-transversality problem into a homotopy lifting problem, thus permitting the application of standard obstruction theory to the problem. In order to simplify the resulting obstructions, we consider in detail only the stable case. The obstruction groups are then Bredon cohomology groups of $M$ with local coefficients. We apply these methods also to the study of near $G$-transversality, obtaining stable obstructions for this case. These obstructions reside, roughly, in Bredon cohomology of $\left(M, M-f^{-1}(Y)\right)$ with local coefficients and map to the global obstructions. If $f$ is not pathological (for example, if $f$ is smooth or PL) then the obstruction groups are precisely the Bredon cohomology of $\left(M, M-f^{-1}(Y)\right)$.

This paper is organized as follows. In $\S 2$ we express the stable $G$-transversality problem in terms of the geometric/homotopical normal bordism dichotomy. In $\S 3$ we express both the unstable and stable $G$-transversality problems as homotopy lifting problems and we relate this to the approach in $\S 2$. In $\S 4$ we obtain the obstructions to stable $G$-transversality and in $\S 5$ we do the same for near $G$-transversality.

\section{A SIMPLE OBSERVATION}

We start this section with some general definitions. Let $Y$ be a compact $G$-space and let $\xi$ be a $G$-vector bundle over $Y$ with Thom space $T \xi$. If $M$ is a compact $G$-manifold, then we say that a $G$-map $f: M \rightarrow T \xi$ is transverse to $Y$ if it is transverse in the usual nonequivariant sense. Similarly, if $M$ has boundary it is clear what we mean if we say that a base point-preserving $G$-map $f: M / \partial M \rightarrow T \xi$ is transverse to $Y$.

We can stabilize as follows. If $W$ is a finite-dimensional orthogonal representation of $G$, let $D(W)$ be its unit disk, let $S(W)=\partial D(W)$, and let $S^{W}=D(W) / S(W)$. We write $\Sigma^{W} X$ for $X \wedge S^{W}$.

Definition 2.1. Let $(M, \partial M)$ be a compact $G$-manifold, and let $\xi$ be a $G$ vector bundle over the compact $G$-space $Y$. A $G$-map $f: M / \partial M \rightarrow T \xi$ can be made stably transverse to $Y$ if there exists a $G$-representation $W$ such that $\Sigma^{W} f: \Sigma^{W} M / \partial M \rightarrow T(\xi \oplus W)$ is $G$-homotopic to a map transverse to $Y$. lem.

In this section we shall translate this problem into a normal cobordism prob-

Definition 2.2. Let $(M, \partial M)$ be a compact $G$-manifold of dimension $m$. Let $(Y, B)$ be a pair of $G$-spaces, and let $\xi$ be a $G$-vector bundle over $Y$ of dimension $k$. Let $\Omega_{n}^{G}(M ; Y, B, \xi)$ be the following bordism group. Consider $(n+m-k)$-dimensional $G$-manifolds $\left(X ; \partial_{0} X, \partial_{1} X\right)$ embedded in $\left(M \times D\left(W \oplus \mathbb{R}^{n}\right) ; \partial M \times D\left(W \oplus \mathbb{R}^{n}\right), M \times S\left(W \oplus \mathbb{R}^{n}\right)\right)$ for some $W$, together with maps $\left(X, \partial_{1} X\right) \rightarrow(Y, B)$ covered by bundle maps $\nu \rightarrow \xi \oplus W$, where $\nu$ is the normal bundle of $X$ in $M \times D\left(W \oplus \mathbb{R}^{n}\right) . \Omega_{n}^{G}(M ; Y, B, \xi)$ is then the group of normal bordism classes of such manifolds (see [B] or [Wal] for 
discussions of normal bordism). Define $\Omega_{n}^{G}(M, \partial M ; Y, B, \xi)$ in the same way, except we insist that $\partial_{0} X=\varnothing$. We call this geometric normal bordism.

An easy argument shows that the following are long exact sequences:

$$
\begin{aligned}
\cdots & \rightarrow \Omega_{n}^{G}(M, \partial M ; Y, B, \xi) \rightarrow \Omega_{n}^{G}(M ; Y, B, \xi) \\
& \rightarrow \Omega_{n}^{G}(\partial M ; Y, B, \xi) \rightarrow \Omega_{n-1}^{G}(M, \partial M ; Y, B, \xi) \rightarrow \cdots, \\
\cdots & \rightarrow \Omega_{n}^{G}(M ; B, \xi) \rightarrow \Omega_{n}^{G}(M ; Y, \xi) \\
& \rightarrow \Omega_{n}^{G}(M ; Y, B, \xi) \rightarrow \Omega_{n-1}^{G}(M ; B, \xi) \rightarrow \cdots .
\end{aligned}
$$

In the former sequence, the first map is the obvious one; the second map takes $\left(X ; \partial_{0} X, \partial_{1} X\right)$ to $\left(\partial_{0} X ; \varnothing, \partial_{0} X \cap \partial_{1} X\right) ;$ and the third takes $(X, \partial X) \subset$ $(\partial M \times D(W), \partial M \times S(W))$ to $X \times\left\{\frac{1}{2}\right\} \subset \partial M \times I \subset M$. In the latter sequence, the maps are the usual ones.

If $V$ is a $G$-representation, then there are maps

$$
\sigma: \Omega_{n}^{G}(M ; Y, B, \xi) \rightarrow \Omega_{n+|V|}^{G}(M ;(Y, B) \times(D(V), S(V)), \xi)
$$

and

$$
\sigma: \Omega_{n}^{G}(M, \partial M ; Y, B, \xi) \rightarrow \Omega_{n+|V|}^{G}(M, \partial M ;(Y, B) \times(D(V), S(V)), \xi)
$$

defined by taking $\left(X ; \partial_{0} X, \partial_{1} X\right)$ to $\left(X \times D(V) ; \partial_{0} X \times D(V), \partial_{1} X \times D(V) \cup\right.$ $X \times S(V))$.

As in $[\mathrm{tD}, \mathrm{BH}]$, we make the following

Definition 2.4. Let

$$
\underline{\Omega}_{n}^{G}(M ; Y, B, \xi)=\operatorname{colim}_{V} \Omega_{n+|V|}^{G}(M ;(Y, B) \times(D(V), S(V)), \zeta),
$$

and define $\underline{\Omega}_{n}^{G}(M, \partial M ; Y, B, \xi)$ similarly. We call this homotopical normal bordism.

The following theorem justifies the name. We write $\{X, Y\}_{G}$ for the group of homotopy classes of stable $G$-maps from $X$ to $Y$, i.e.,

$$
\{X, Y\}_{G}=\operatorname{colim}_{V}\left[\Sigma^{V} X, \Sigma^{V} Y\right]_{G} .
$$

Also, if $X$ is a $G$-space, we write $X^{+}$for $X$ with a $G$-fixed disjoint basepoint adjoined.

Proposition 2.5. There are natural isomorphisms

$$
\underline{\Omega}_{n}^{G}(M ; Y, B, \xi) \cong\left\{\Sigma^{n} M^{+}, T \xi / T(\xi \mid B)\right\}_{G}
$$

and

$$
\underline{\Omega}_{n}^{G}(M, \partial M ; Y, B, \xi) \cong\left\{\Sigma^{n} M / \partial M, T \xi / T(\xi \mid B)\right\}_{G} .
$$

Proof. The natural isomorphisms are given by the Pontrjagin-Thom construction. For example, define $\Phi: \underline{\Omega}_{n}^{G}(M ; Y, \xi) \rightarrow\left\{\Sigma^{n} M^{+}, T \xi\right\}_{G}$ as follows. Given the manifold $X \subset M \times D\left(W \oplus \mathbb{R}^{n}\right)$, we take the composite

$$
\Sigma^{W} \Sigma^{n} M^{+} \rightarrow T \nu / T\left(\nu \mid \partial_{1} X\right) \rightarrow T(\xi \oplus W)=\Sigma^{W} T \xi,
$$


where the first map is the collapse and the second is given by the normal map $\nu \rightarrow \xi \oplus W$. Since colimits preserve exact sequences, the sequences (2.3) remain exact if $\Omega$ is replaced with $\underline{\Omega}$. Using these it suffices to prove one case, namely,

$$
\underline{\Omega}_{n}^{G}(M ; Y, \xi) \cong\left\{\Sigma^{n} M^{+}, T \xi\right\}_{G} .
$$

The inverse map, $\Psi:\left\{\Sigma^{n} M^{+}, T \xi\right\}_{G} \rightarrow \underline{\Omega}_{n}^{G}(M ; Y, \xi)$ is defined using transversality: Represent an element of $\left\{\Sigma^{n} M^{+}, T \xi\right\}_{G}$ by a map of pairs

$$
f:\left(M \times D\left(W \oplus \mathbb{R}^{n}\right), M \times S\left(W \oplus \mathbb{R}^{n}\right)\right) \rightarrow(D(\xi \oplus W), S(\xi \oplus W)) .
$$

We can think of $D(\xi \oplus W)$ as $D\left(p^{*} \xi\right)$, where $p^{*} \xi$ is the bundle over $Y \times D(W)$ induced by $\xi$. As such, if $W$ is large enough, then Wassermann's transversality results [Was] imply that $f$ is $G$-homotopic to a map that is transverse to $Y \times$ $D(W)$, since for large enough $W$ there will be an epimorphism from $W$ onto $\xi$. Let $X \subset M \times D\left(W \oplus \mathbb{R}^{n}\right)$ then be the preimage of $Y \times D(W)$, and let $\Psi[f]$ be the normal bordism class of $X$.

Standard arguments now show that $\Psi$ is well defined and inverse to $\Phi$.

Now let us return to the question of stable transversality. Given a map $f: M / \partial M \rightarrow T \xi$ as in Definition 2.1, $f$ determines a class $[f] \in\{M / \partial M, T \xi\}_{G}$ $\cong \underline{\Omega}_{0}^{G}(M, \partial M ; Y, \xi)$. Let $s: \Omega_{n}^{G}(M, \partial M ; Y, \xi) \rightarrow \underline{\Omega}_{n}^{G}(M, \partial M ; Y, \xi)$ be the canonical map.

Theorem 2.6. $f$ can be made stably transverse to the $G$-map $Y$ iff $[f] \in$ $\underline{\Omega}_{0}^{G}(M, \partial M ; Y, \xi)$ is in the image of $s$.

Thus, we can view the obstruction to $f$ being stably transverse to $Y$ as the image of $[f]$ to $\underline{\Omega}_{0}^{G}(M, \partial M ; Y, \xi) / s \Omega_{0}^{G}(M, \partial M ; Y, \xi)$.

Proof. If $f$ can be made stably transverse to $Y$, then there exists a $W$ for which the map $(M \times D(W), M \times S(W)) \rightarrow(D(\xi \oplus W), S(\xi \oplus W))$ is $G$ homotopic to a map transverse to $Y$. If we take $X$ to be the preimage of $Y$, then $X$ and its normal data define an element of $\underline{\Omega}_{0}^{G}(M, \partial M ; Y, \xi)$ whose image under $s$ is $[f]$.

Conversely, maps in the image of $s$ are clearly transverse to $Y$.

\section{A LIFTING PROBLEM}

We now show how the transversality problem can be recast as a lifting problem.

We will use the following general situation in the rest of the paper. Consider a compact $G$-space $Y$ and a $G$-vector bundle $\xi$ over $Y$. Suppose that $N$ is a $G$-space containing $Y$ as a subspace, such that $Y$ has a closed $G$-invariant neighborhood $D$ in $N$ homeomorphic to $D(\xi)$. In particular, $N$ might be $T \xi$, or $Y$ might be a submanifold of a $G$-manifold $N$; these are the cases of most interest.

If $M$ is a compact $G$-manifold, then it is clear what is meant for a $G$-map $f: M \rightarrow N$ to be transverse to $Y$. The unstable $G$-transversality problem is this: Given a $G$-map $f: M \rightarrow N$ that is transverse to $Y$ on a $G$-invariant subspace $A \subset M$, is $f$ homotopic rel $A$ a map that is transverse to $Y$ on $M$ ?

Assume for the moment that $f$ is transverse to $Y$. By definition, $f$ induces a $G_{x}$-equivariant epimorphism from the tangent space at each point $x$ 
in $f^{-1}(Y)$ to $\xi_{f(x)}$, the fiber of $\xi$ over $f(x)$. This continues to hold in a tubular neighborhood of $Y$ in $N$. Precisely, there is an $r$ with $0<r \leq 1$ such that, if $U=\operatorname{Int} D_{r}(\xi) \subset D$ is the interior of the disc bundle of radius $r$ then at each $x \in f^{-1}(U)$ the map $d f: \tau M_{x} \rightarrow \xi_{\pi(f(x))}$ is epic; here $d f$ denotes the derivative of $f$ in the direction of the fibers of $\xi$ and $\pi: \xi \rightarrow Y$ denotes the projection. If we change $f$ by a homotopy, we can assume that $r=1$. This motivates the following construction.

Definition 3.1. We define a $G$-space $\overline{E p i}(M ; N, Y)$, or $\overline{E p i}$ when $M, N$, and $Y$ are clear, as $E \cup_{C} F$, where

$$
\begin{aligned}
& E=\left\{(m, n, \phi): m \in M, n \in D, \phi: \tau M_{m} \rightarrow \xi_{\pi(n)} \text { a linear epimorphism }\right\}, \\
& C=\{(m, n, \phi) \in E: n \in \partial D\}, \\
& F=M \times(N-\operatorname{Int} D),
\end{aligned}
$$

and $f: C \rightarrow F$ is the obvious map.

Let $p: \overline{E p i} \rightarrow M$ and $q: \overline{E p i} \rightarrow N$ be the projections. Note that $p$ is a fiber bundle whose group can be taken to be $O(m)$ where $m=\operatorname{dim} M$. Indeed, the associated principal bundle is isomorphic with that of $\tau M$. Although $q$ is not a bundle, its restriction to $q^{-1}(\operatorname{Int} D)$ is a $G$-bundle with principal bundle agreeing with that of $\pi^{*}(\xi)$.

The discussion before the definition shows that if the $G$-map $f: M \rightarrow N$ is transverse to $Y$, then, for suitable choice of $D$ or by changing $f$ by a $G$ homotopy through transverse maps, $d f$ gives a lift $\theta$ making the following diagram commute:

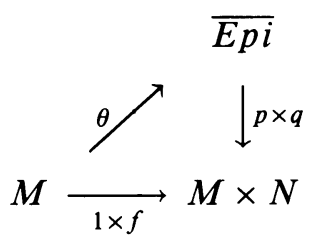

We can think of this as a diagram over $M$ in the obvious way. Also note that, if $f$ is only transverse to $Y$ on $A \subset M$, then $\theta$ will only be defined on $A$.

The following theorem shows that the transversality problem is equivalent to a lifting problem.

Theorem 3.3. Suppose that $f: M \rightarrow N$ is transverse to $Y$ on $A$ with associated lift $\theta_{0}$ defined on $A$. Then $f: M \rightarrow N$ is G-homotopic rel $A$ to a map transverse to $Y$ iff there exists a lift $\theta$ extending $\theta_{0}$ making diagram (3.2) G-homotopy commute over $M \operatorname{rel} A$.

Proof. We have already shown one direction. As to the converse, assume that we have a $\theta$ making diagram (3.2) $G$-homotopy commute over $M \operatorname{rel} A$. Since $q \theta \simeq f$ rel $A$, we can assume, by replacing $f$ with $q \theta$, that the diagram strictly commutes.

Before continuing with the proof, we recall a concept due to Wassermann [Was]. Assume that $C \subset M$ is a $G$-invariant subspace and that we are given $\theta$ such that diagram (3.2) commutes. Then we say that $f$ is $\theta$-transverse to $Y$ on $C$ if:

(1) $f$ is transverse to $Y$ on $C$; 
(2) If $c \in C \cap f^{-1}(Y)$ and we use the exponential map to identify a neighborhood of 0 in $\tau M_{c}$ with a neighborhood of $c$ in $M$, then the restriction of $f$ to the orthogonal complement of the $G_{c}$-fixed set is linear and agrees with $\theta$.

We now mimic Wasserman's argument to show that the presence of $\theta$ allows us to $G$-homotope $f$ to a map transverse to $Y$. The argument proceeds by induction over fixed sets, the inductive hypothesis being the following: If $H \subset$ $G$, let $M^{>H}$ be the union of proper fixed subspaces of $G M^{H}=M^{(H)}$, and assume by induction that we have $G$-homotopies $f \simeq f^{\prime}$ and $\theta \simeq \theta^{\prime}$ rel $A$ such that $(p \times q) \circ \theta^{\prime}=1 \times f^{\prime}$ and $f^{\prime}$ is $\theta^{\prime}$-transverse on $M^{>H}$. We can now $G$-homotope $f^{\prime}$ to a map $f^{\prime \prime}$ that is transverse to $Y^{(H)}$ on $M^{(H)}$; this is a nonequivariant problem. Since we can accomplish this with a small homotopy, ensuring that no tracks leave or enter the neighborhood $D$ of $Y$, we can lift this homotopy over $p \times q$ to obtain $\theta^{\prime \prime} \simeq \theta^{\prime}$. We now use $\theta^{\prime \prime}$ and Wasserman's arguments to make $f^{\prime \prime} \theta^{\prime \prime}$-transverse to $Y$ on $M^{(H)}$, thus completing the inductive step.

Turning to stable transversality, assume that $N$ has a base point outside of $D$. As an immediate consequence of Theorem 3.3 we have

Corollary 3.4. $f: M / A \rightarrow N$ can be made stably transverse to $Y$ iff there exists a $W$ for which there exists a G-homotopy lift $\theta$ over $\Sigma^{W} M / A$ rel base point in the following diagram:

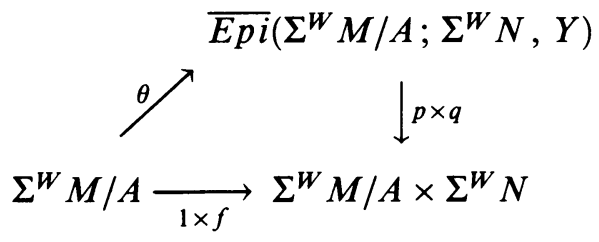

Here $\overline{E p i}$ is defined as before, except that over $* \times n$ we have just a single point, even if $n \in D$.

The spaces that appear in diagram (3.5) are the $W$ th spaces of prespectra. In the bottom row we see the suspension spectrum $\Sigma^{\infty} M / A$ and the product spectrum $\Sigma^{\infty} M / A \times \Sigma^{\infty} N$ (see [LMS, §1] for generalities about equivariant spectra and prespectra). The top space is the $W$ th space of a prespectrum whose associated spectrum we shall call $E=E(M / A ; N, Y)$. The structure map

$$
\Sigma^{Z} \overline{\operatorname{Epi}}\left(\Sigma^{W} M / A ; \Sigma^{W} N, Y\right) \rightarrow \overline{E p i}\left(\Sigma^{W+Z} M / A ; \Sigma^{W+Z} N, Y\right)
$$

is given by sending $(m, n, \phi, z)$ to $((m, z),(n, z), \phi+\mathrm{id})$ for $z \in S^{Z}$. Thus we can restate Corollary 3.4 as follows: $f: M / A \rightarrow N$ can be made stably transverse to $Y$ iff there exists a map of spectra $\theta$ making the following diagram homotopy commute over $\Sigma^{\infty} M / A$ :

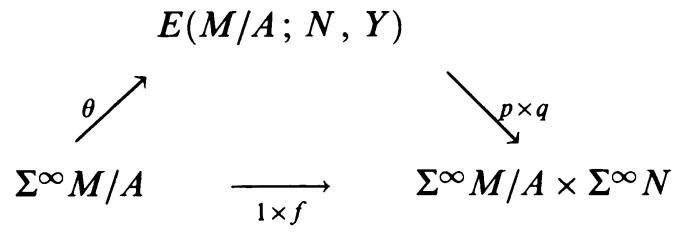

We can now relate these results to those of $\S 2$. Let $h \Gamma_{n}(E)$ be the set of $G$-homotopy classes of sections of $p: E\left(\Sigma^{n} M / \partial M ; T \xi, Y\right) \rightarrow \Sigma^{n} \Sigma^{\infty} M / \partial M$. 
Then there is a split surjection $\Psi: h \Gamma_{n}(E) \rightarrow \Omega_{n}^{G}(M, \partial M ; Y, \xi)$ given by transversality, using Corollary 3.4. A left inverse $\Psi: \Omega_{n}^{G}(M, \partial M ; Y, \xi) \rightarrow$ $h \Gamma_{n}(E)$ of $\Psi$ is given by the Pontrjagin-Thom construction, but $\Psi$ fails to be an isomorphism even in the nonequivariant case. While we suspect that $E$ could be modified to make $\Psi$ an isomorphism, this would unnecessarily complicate the obstruction theory to follow. However, both $\Omega_{n}^{G}(M, \partial M ; Y, \xi)$ and $h \Gamma_{n}(E)$ map to $\underline{\Omega}_{n}^{G}(M, \partial M ; Y, \xi)$, and it follows that the two cokernels coincide. Thus the obstruction defined in Theorem 2.6 may be regarded as an element of $\underline{\Omega}_{0}^{G}(M, \partial M ; Y, \xi) / h \Gamma_{0}(E)$, and thus is the obstruction to the existence of a homotopy lift $\theta$ in diagram (3.6).

\section{OBSTRUCTIONS TO STABLE $G$-TRANSVERSALITY}

The obstruction $v$ to stable $G$-transversality has been shown in Theorem 2.6 to be an element of a certain cokernel; however, the groups involved are difficult to compute. As pointed out at the end of $\S 3$, we may identify $v$ with the obstruction to a lifting problem. Thus we can use standard obstruction theory to replace $v$ with a series of simpler obstructions. In order to define the latter, we first need to recall the notion of Bredon cohomology with local coefficients.

Definition 4.1. The fundamental groupoid $\pi(X ; G)$ of the $G$-space $X$ is the following category. For the objects, we take the $G$-maps $x: G / H \rightarrow X$ (or equivalently, points $x \in X^{H}$ ) for subgroups $H \subset G$. A morphism from $x$ to $y: G / K \rightarrow X$ in $\pi(X ; G)$ is an equivalence class $[\omega, \sigma]$, where $\sigma: G / H \rightarrow$ $G / K$ is a $G$-map and $\omega$ is a $G$-homotopy $x \simeq y \circ \sigma .(\omega, \sigma) \approx\left(\omega^{\prime}, \sigma\right)$ if $\omega$ and $\omega^{\prime}$ are homotopic rel end points. Note that $\pi(X ; G)$ comes equipped with a covariant functor $\varphi: \pi(X ; G) \rightarrow \mathscr{G}$, where $\mathscr{G}$ is the category of $G$-orbits $G / H$ and $G$-maps, viz. $\varphi(x: G / H \rightarrow X)=G / H$ and $\varphi[\omega, \sigma]=\sigma$.

A local coefficient system on $X$ is then a contravariant functor $T: \pi(X ; G) \rightarrow$ $\mathscr{A} b$ where $\mathscr{A} b$ is the category of abelian groups. Given a $G$-space $X$ and a local coefficient system $T$ on $X$, one can construct Bredon cohomology with local coefficients [MS, M].

Consider again the general setting for stable $G$-transversality. Thus we are given a $G$-map $f: M / A \rightarrow N$ and $Y \subset N$ as described at the beginning of $\S 3$. By $\S 4, f$ can be made stably transverse to $Y$ iff there exists a map of spectra $\theta$ making the following diagram homotopy commute over $\Sigma^{\infty} M / A$ :

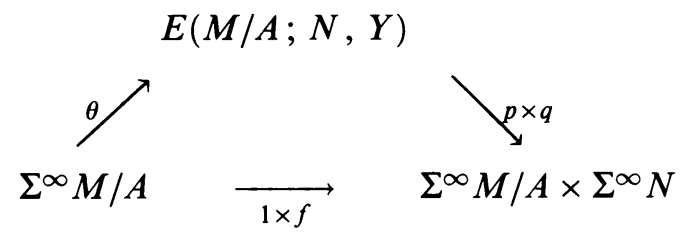

Let $M^{n}$ denote the relative $n$-skeleton of $(M, A)$, assume that $\theta$ has been constructed on $\Sigma^{\infty} M^{n} / A$, and consider the obstruction to extending $\theta$ over $\Sigma^{\infty} M^{n+1} / A$. The map $\theta$ can be extended over a cell of the form $G / H \times D^{n+1}$ in $M^{n+1}$ iff the associated $H$-map

$$
(D(W+n+1), S(W+n+1)) \rightarrow\left(\Sigma^{W} M / A \times \Sigma^{W} N, \overline{\operatorname{Epi}}\left(\Sigma^{W} M / A ; \Sigma^{W} N, Y\right)\right)
$$


is equivariantly trivial over $\Sigma^{W} M / A$ for some $W$. Since $\tau M$ is trivial over the cell in question, we can replace $\overline{\operatorname{Epi}}\left(\Sigma^{W} M / A ; \Sigma^{W} N, Y\right)$ with $\overline{E p i}\left(\tau M_{m} \oplus W ; \Sigma^{W} N, Y\right) \times D(W+n+1)$, where $m$ is the center of $\{e H\} \times D^{n+1}$ and where $\overline{E p i}\left(F ; \Sigma^{W} N, Y\right)$ is defined in the same way as $\overline{\operatorname{Epi}}(M ; N, Y)$, except that in place of $M$ we use a single point and in place of $\tau M$ we use the single $H$-vector space $F$. Since we are now lifting to the total space of a product bundle, we can represent this local lifting problem as the relative stable $H$-homotopy class of

$$
(D(W+n+1), S(W+n+1)) \rightarrow\left(\Sigma^{W} N, \overline{E p i}\left(\tau M_{m} \oplus W ; \Sigma^{W} N, Y\right)\right),
$$

or as an element of $\pi_{n+1}^{H}\left(\Sigma^{\infty} N, E\left(\tau M_{m} ; N, Y\right)\right)$, where $E\left(\tau M_{m} ; N, Y\right)$ is the $H$-spectrum whose $W$ th space is $\overline{E p i}\left(\tau M_{m} \oplus W ; \Sigma^{W} N, Y\right)$. With $q_{m}(N, Y)$ : $E\left(\tau M_{m} ; N, Y\right) \rightarrow \Sigma^{\infty} N$ denoting the projection, we can replace $\pi_{n+1}^{H}\left(\Sigma^{\infty} N, E\left(\tau M_{m} ; N, Y\right)\right)$ by $\pi_{n}^{H}\left(C q_{m}(N, Y)\right)$, where $C q_{m}$ is the cofiber of $q_{m}$.

We can now use this to define a local coefficient system $\pi_{n}\left(C q_{\#}(N, Y)\right)$ on $M$ by taking $\pi_{n}\left(C q_{*}(N, Y)\right)(x: G / H \rightarrow M)=\pi_{n}^{H}\left(C q_{x(e H)}(N, Y)\right)$. The action of $\pi_{n}\left(C q_{\#}(N, Y)\right)$ on morphisms is given by the $G$-covering homotopy property for $\tau M$. Standard arguments now give us

Theorem 4.2. $A$ G-map $f: M / A \rightarrow N$ can be made stably transverse to $Y$ iff a series of obstructions $v_{n+1}$ in $H_{G}^{n+1}\left(M / A ; \pi_{n}\left(C q_{\#}(N, Y)\right)\right)$ vanish.

A rather surprising consequence of the theorem is that the obstructions depend only on the Thom space of $\xi$ (recall that the disc bundle of $\xi$ is a subspace of $N)$.

Corollary 4.3. (a) $A$ G-map $f: M / A \rightarrow N$ can be made stably transverse to $Y$ iff a series of obstructions $v_{n+1}$ in $H_{G}^{n+1}\left(M / A ; \pi_{n}\left(C q_{\#}(T \xi, Y)\right)\right)$ vanish.

(b) Let $c: N \rightarrow T \xi$ be the collapse. Then $f: M / A \rightarrow N$ can be made stably transverse to $Y$ iff $c \circ f: M / A \rightarrow T \xi$ can.

Proof. For (a), note that $c: N \rightarrow T \xi$ induces a map $\overline{E p i}\left(\tau M_{m} \oplus W ; \Sigma^{W} N, Y\right)$ $\rightarrow \overline{E p i}\left(\tau M_{m} \oplus W ; \Sigma^{W} T \xi, Y\right)$ and that, in the diagram

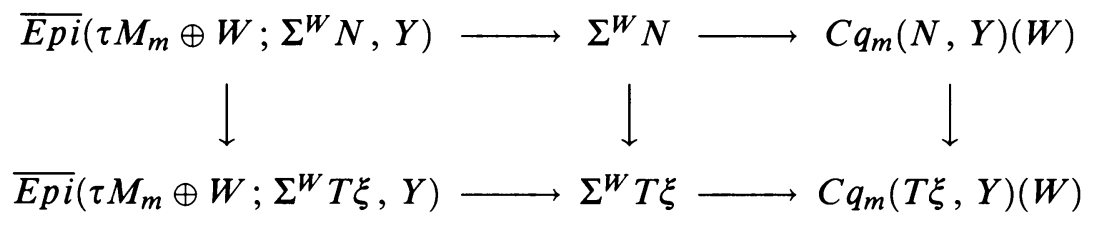

one can easily see that the map $C q_{m}(N, Y)(W) \rightarrow C q_{m}(T \xi, Y)(W)$ is given by collapsing out a contractible subspace. In essence, this is excision for the relative homotopy groups. Part (b) now follows formally, since $c$ induces an isomorphism of obstruction groups and takes obstructions to obstructions.

Remarks 4.4. (a) The space $\overline{E p i}\left(\tau M_{m} \oplus W ; \Sigma^{W} T \xi, Y\right)$ may in fact be described as the Thom space of an $H$-vector bundle over the space $\operatorname{Epi}\left(\tau M_{m} \oplus W, \xi \oplus W\right)$ of linear epimorphisms. Let $r: E p i\left(\tau M_{m} \oplus W, \xi \oplus W\right) \rightarrow Y$ be the bundle projection. Then $\overline{E p i}\left(\tau M_{m} \oplus W ; \Sigma^{W} T \xi, Y\right) \cong T\left(r^{*} \xi \oplus W\right)$. This fact, together with Corollary 4.3(a), allows us to view the coefficient system as a relative normal bordism group. On the other hand, Corollary 4.3(b) together with Theorem 2.6 allows us to view the global obstruction in terms of relative normal bordism. 
(b) Assume that for each $H \subset G$, each component $C$ of $M^{H}$ has dimension lower than the connectivity of $\operatorname{Epi}\left(\tau M_{m} \oplus W, \xi_{y} \oplus W\right)^{K}$ for some $W$, with $m \in C$, whenever $K \subset H$ and $y \in Y^{K}$. This connectivity can be computed as follows, as in [P, II.4.6]. Decompose $\tau M_{m}-\xi_{y}$ as a sum of $K$ irreducibles; $\tau M_{m}-\xi_{y}=\mathbb{R}^{n_{0}} \oplus \sum Z_{i}^{n_{i}}$. Let $d_{i}=1,2$, or 4 , if $Z_{i}$ is real, complex, or quaternionic, respectively $\left(d_{0}=1\right)$. Then the connectivity of $\operatorname{Epi}\left(\tau M_{m} \oplus W, \xi_{y} \oplus W\right)^{K}$ is $\min _{i}\left\{d_{i}\left(n_{i}+1\right)-2\right\}$. By Remark (a) above, the coefficient groups $\pi_{n}\left(C q_{\#}(T \xi, Y)\right)$ vanish if the dimension of $C$ is less than this connectivity. Note that this condition is essentially the condition needed to ensure that the coefficients vanish in Petrie's formulation [P, II.4].

\section{Obstructions to STABLE NEAR $G$-TRANSVERSALITy}

We now turn to the obstruction theory for "near" $G$-transversality. To make sense of this, we assume that $N$ is a metric space such that $D=\bar{B}(Y, 1)$.

Definition 5.1. We say that $f: M / A \rightarrow N$ is nearly $G$-transverse to $Y$ if for each $\varepsilon>0, f$ can be made $G$-homotopic to a transverse map through an $\varepsilon$-homotopy. Here, an $\varepsilon$-homotopy is one in which the diameter of each track is less than $\varepsilon$.

Before seeking conditions under which $f$ is nearly $G$-transverse to $Y$, we must be a little more fastidious with the construction of $\overline{E p i}$. If $\delta>0$, let $\overline{E p i}_{\delta}$ be constructed in the same way as was $\overline{E p i}$ except that $D$ is replaced by $\bar{B}(Y, \min \{1, \delta\})$. By the theory in $\S 3, f$ is nearly transverse to $Y$ iff $f$ nearly lifts to $\overline{E p i}$, i.e., for each $\varepsilon>0$ there is a $\delta>0$ and a lift $\theta$ making diagram (3.2) $\varepsilon$-homotopy commute over $M$, where $\overline{E p i}$ is replaced by $\overline{E p i}_{\delta}$. This follows because, once we have $\theta$, the argument of Theorem 3.3 involves arbitrarily small homotopies.

We now proceed as follows. Let $0<\varepsilon<1$ be so small that the preimage under $\pi: D \rightarrow Y$ of any set of diameter $\varepsilon$ is a product. Choose a finite open cover of $Y$ in $N$ by products of the form $U_{y}=B_{Y}(y, \varepsilon / 2) \times B_{F}(y, \varepsilon / 2)$, where $F=\pi^{-1}(y)$. We can also assume that $G B_{Y}(y, \varepsilon / 2) \cong G \times_{G_{y}} B_{Y}(y, \varepsilon / 2)$. Call any $G$-triangulation of $M$ subordinate of $\left\{f^{-1}\left(U_{y}\right), f^{-1}(N-Y)\right\}$ an $\varepsilon$ triangulation. Consider the following condition on $f$ :

Condition 5.2. For each $\varepsilon>0$ and each $\varepsilon$-triangulation there exists a $\delta>0$, an $\varepsilon$-homotopy $h: f \simeq f^{\prime}$ and a lift $\theta$ of $f^{\prime}$ to $\overline{E p i}_{\delta}$ such that if $\sigma$ is a simplex in $M$ with $f(\sigma) \subset U_{y}$ then $h(\sigma \times I) \subset U_{y}$.

Lemma 5.3. $f: M / A \rightarrow N$ is nearly transverse to $Y$ iff Condition 5.2 holds.

Proof. Sufficiency is clear. For the other direction, assume that $f$ is almost transverse to $Y$, let $\varepsilon>0$ be as above, and assume given an $\varepsilon$-triangulation of $M$. Choose $\varepsilon^{\prime} \leq \varepsilon$ such that $B\left(f(\sigma), \varepsilon^{\prime}\right) \subset U_{y}$ whenever $f(\sigma) \subset U_{y}$. By the hypothesis, there is an $\varepsilon^{\prime}$-homotopy of $f$ to $f^{\prime}$ and a lift of $f^{\prime}$ to some $\overline{E p i}_{\delta}$. But the choice of $\varepsilon^{\prime}$ ensures that the latter part of Condition 5.2 holds.

We now consider the obstructions to stably nearly lifting $f$. Let $\varepsilon>0$ and assume we are given an $\varepsilon$-triangulation of $M$. Let $M^{n}$ denote the relative $n$ skeleton of $(M, A)$ and assume inductively that $f$ stably nearly lifts on $M^{n}$. Let $\varepsilon^{\prime}<\varepsilon / 6$ be small enough so that $f(\sigma)$ is at least $2 \varepsilon^{\prime}$ from $Y$ if $\sigma$ is a simplex such that $f(\sigma) \cap Y=\varnothing$. Choose an $\varepsilon^{\prime}$-homotopy $h: f \mid M^{n} \times D(W) \simeq f^{\prime}$ 
and a lift $\theta$ of $f^{\prime}$ to some $\overline{E p i}_{\delta}$ with $\delta<\varepsilon^{\prime}$, satisfying the latter part of Condition 5.2 in the following form: if $\sigma$ is a simplex in $M^{n}$ with $f(\sigma) \subset U_{y}$ then $h\left(\sigma \times D_{\varepsilon / 3}(W) \times I\right) \subset U_{y} \times D_{\varepsilon / 2}(W)$. We seek to extend $h$ over $M^{n+1}$ satisfying Condition 5.2 in the same way. As seen earlier, the obstruction to extending $h$ and $\theta$ over a cell of the form $G / H \times \sigma^{n+1}$ in $M^{n+1}$ is the stable $H$-equivariant homotopy class of an $H$-map

$$
(D(W+n+1), S(W+n+1)) \rightarrow\left(\Sigma^{W} N, \overline{E p i}_{\delta}\left(\tau M_{m} \oplus W ; \Sigma^{W} N, Y\right)\right) .
$$

Also note that, by our choice of $\varepsilon^{\prime}$ and the restrictions on $h$, if $f(\sigma) \cap Y=\varnothing$ then $h(\partial \sigma \times D(W) \times I) \cap \bar{B}(Y, \delta)=\varnothing$. Further, $h\left(\partial \sigma \times\left(D(W)-D_{\varepsilon / 3}(W)\right) \times I\right)$ $\cap \bar{B}(Y, \delta)=\varnothing$ for all $\sigma$. In view of this, there is no obstruction to extending $h$ and $\theta$ over $\sigma \times D(W)$ if $f(\sigma) \cap Y=\varnothing$, and the same is true for $\sigma \times(D(W)-$ $\left.D_{\varepsilon / 3}(W)\right)$ for all $\sigma$. Thus we consider the obstruction to extending $h$ and $\theta$ over $\sigma \times D_{\varepsilon / 3}(W)$, where $f(\sigma)$ meets $Y$. This is the $H$-homotopy class of an $H$-map

$$
\begin{aligned}
& (D(W+n+1), S(W+n+1)) \\
& \quad \rightarrow\left(U_{y} \times D_{\varepsilon / 2}(W), \overline{E p i}_{\delta}\left(\tau M_{m} \oplus W ; U_{y} \times D_{\varepsilon / 2}(W), Y \cap U_{y}\right)\right)
\end{aligned}
$$

for some $y \in Y^{H}$. Since $U_{y}$ is a product neighborhood, this is equivalent to the $H$-homotopy class of an $H$-map

$$
(D(W+n+1), S(W+n+1)) \rightarrow\left(D\left(\xi_{y} \oplus W\right), \overline{E p i}_{\delta}\left(\tau M_{m} \oplus W ; D\left(\xi_{y} \oplus W\right), 0\right)\right) .
$$

Passing to the colimit over $W$ gives an element of $\pi_{n+1}^{H}\left(*, E\left(\tau M_{m} ; D\left(\xi_{y}\right), 0\right)\right)$ $\cong \pi_{n}^{H}\left(E\left(\tau M_{m} ; D\left(\xi_{y}\right), 0\right)\right)$. Here, $E\left(\tau M_{m} ; D\left(\xi_{y}\right), 0\right)$ is the $H$-spectrum whose $(\mathbb{R} \oplus W)$ th space is $\overline{E p i}\left(\tau M_{m} \oplus \mathbb{R} \oplus W ; \Sigma^{W} D\left(\xi_{y} \oplus \mathbb{R}\right), 0\right)$, where we base $D\left(\xi_{y} \oplus \mathbb{R}\right)$ at $(0,1)$.

Let $C \subset M$ be the $G$-subcomplex consisting of all simplices $\sigma$ with $f(\sigma) \cap$ $Y=\varnothing$. Define a local coefficient system $\pi_{n}\left(E\left(\tau M_{\#} ; D\left(\xi_{f(\#)}\right), 0\right)\right)$ on $M-C$ by taking

$$
\pi_{n}\left(E\left(\tau M_{\#} ; D\left(\xi_{f(\#)}\right), 0\right)\right)(x: G / H \rightarrow M)=\pi_{n}^{H}\left(E\left(\tau M_{x(e H)} ; D\left(\xi_{f(x(e H))}\right), 0\right)\right) .
$$

The action on morphisms is given by the $G$-covering homotopy property for $\tau M$ and $\xi$.

The discussion so far gives obstruction elements in the cohomology groups $H_{G}^{n+1}\left(M, C ; \pi_{n}\left(E\left(\tau M_{\#} ; D\left(\xi_{f(\#)}\right), 0\right)\right)\right)$. Here, the subcomplex $C$ depends on the choice of $\varepsilon$ and the particular triangulation used. Define $\mathscr{K}_{G}^{n+1}(M, U)=$ $\lim H_{G}^{n+1}(M, C)$, the limit running over all $G$-triangulations of $M$ and $G$ subcomplexes $C \subset U$. Equivalently, by cofinality, we can take the limit over $G$-invariant compact subsets of submanifolds of $U$. When $f^{-1}(Y)$ is "nice," for example, a $G$-subcomplex of some triangulation $M$, then $\mathscr{H}_{G}^{n+1}\left(M, M-f^{-1}(Y)\right)=H_{G}^{n+1}\left(M, M-f^{-1}(Y)\right)$. The discussion of the preceding paragraphs, together with standard subdivision arguments, shows the following.

Theorem 5.4. $A$ G-map $f: M / A \rightarrow N$ is stably nearly $G$-transverse to $Y$ iff $a$ series of obstructions $v_{n+1}$ in $\mathscr{H}_{G}^{n+1}\left(M, M-f^{-1}(Y) ; \pi_{n}\left(E\left(\tau M_{\#} ; D\left(\xi_{f(\#)}\right), 0\right)\right)\right)$ vanish. 
As in Remark 4.4(b), we can write down sufficient conditions for these obstruction groups to vanish. If $V$ and $W$ are orthogonal $G$-vector spaces, recall that $\operatorname{Epi}(V, W)$ is the $G$-space of linear epimorphisms $V \rightarrow W$. Let $U$ be an open invariant neighborhood of $f^{-1}(Y)$ contained in $f^{-1}(D)$. If $m \in U^{H}$, let $n(m)$ be the smallest connectivity of $\operatorname{Epi}\left(\tau M_{m}, \xi_{f(m)}\right)^{K}$, taken over all $K \subset H$, as computed in Remark 4.4(b). Note that $n(m)$ depends only on the component of $m$ in $U^{H}$, and we shall write $n_{C}$ for $n(m)$ if $C$ is the component of $U^{H}$ containing $m$. We now have

Corollary 5.5. Assume that for each $H \subset G$ and each component $C$ of $U^{H}$ one has $\operatorname{dim} C<n_{C}$. Then $f$ is stably nearly $G$-transverse to $Y$.

Proof. Let $y=f(m)$. We show that the coefficient groups

$$
\pi_{n}\left(E\left(\tau M_{m} ; D\left(\xi_{f(m)}\right), 0\right)\right)
$$

vanish. Consider the Puppe sequence

$$
\begin{aligned}
S\left(\xi_{y} \oplus W\right) & \rightarrow \overline{E p i}\left(\tau M_{m} \oplus W ; D\left(\xi_{y} \oplus W\right), 0\right) \\
& \rightarrow E p i\left(\tau M_{m} \oplus W, \xi_{y} \oplus W\right)_{+} \wedge S^{\xi_{y} \oplus W} \rightarrow \cdots
\end{aligned}
$$

By the hypothesis, the connecting map in equivariant stable homotopy is an isomorphism through dimension $n(m)$ and an epimorphism in dimension $n(m)+$ 1 . Indeed, the fact that $n(m)$ must be positive if the hypothesis is to hold ensures that $\operatorname{Epi}\left(\tau M_{m} \oplus W, \xi_{y} \oplus W\right)^{H} \neq \varnothing$, showing that

$$
\operatorname{Epi}\left(\tau M_{m} \oplus W, \xi_{y} \oplus W\right)_{+} \wedge S^{\xi_{y} \oplus W} \rightarrow S^{\xi_{y} \oplus W}
$$

splits.

Remark 5.6. There is a natural homomorphism

$$
\begin{aligned}
\gamma: & \mathscr{K}_{G}^{n+1}\left(M, M-f^{-1}(Y) ; \pi_{n}\left(E\left(\tau M_{\#} ; D\left(\xi_{f(\#)}\right), 0\right)\right)\right) \\
& \rightarrow H_{G}^{n+1}\left(M / A ; \pi_{n}\left(C q_{\#}(N, Y)\right)\right)
\end{aligned}
$$

given by the restriction map on the arguments and by the inclusion of $D$ in $N$ on coefficients. The similarity of our constructions shows that $\gamma$ carries the obstructions to stable near $G$-transversality to the obstructions to stable $G$-transversality.

\section{REFERENCES}

[B] W. Browder, Surgery on simply-connected manifolds, Springer-Verlag, Berlin, 1972.

[BH] T. Brocker and E. C. Hook, Stable equivariant bordism, Math. Z. 129 (1972), 269-277.

[CW] S. R. Costenoble and S. Waner, The equivariant Spivak normal bundle and equivariant surgery, Michigan Math. J. (to appear).

[tD] T. tom Dieck, Bordims of G-manifolds and integrality theorems, Topology 9 (1970), 345358.

[LMS] L. G. Lewis, Jr., J. P. May, and M. Steinberger, with contributions by J. E. Mc Clure, Equivariant stable homotopy theory, Lecture Notes in Math., vol. 1213, Springer-Verlag, Berlin, 1986.

[MS] I. Moerdijk and J.-A. Svensson, The equivariant Serre spectral sequence, preprint, University Utrecht, 1990.

[M] J. M. Møller, On equivariant function spaces, Pacific J. Math. 142 (1990), 103-119. 
[P] T. Petrie, Pseudoequivalences of G-manifolds, Proc. Sympos. Pure Math., vol. 32, Amer. Math. Soc., Providence, RI, 1978, pp. 169-210.

[Wal] C. T. C. Wall, Surgery on compact manifolds, Academic Press, London, 1970.

[Was] A. G. Wasserman, Equivariant differential topology, Topology 8 (1969), 127-150.

Department of Mathematics, Hofstra University, Hempstead, New York 11550 E-mail address: MATSRC@HOFSTRA.BITNET 